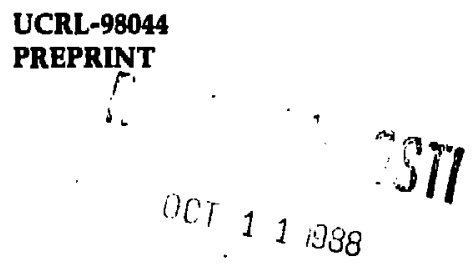

\title{
A Fast Radiation-to-Coherent Light Converter
}

\author{
C. L. Wang, J. E. Flatley, P. H. Stewart, \\ N. Bar-Chaim, K. Y. Lau, I. Ury, \\ R. M. Baltrusaitis, T. J. Davies, B. A. Davis, \\ S. G. Iversen, B. R. Marshall, M. C. Thomas, \\ and P. A. Zagarino
}

This paper was prepared for submittal to SPIE's O-E/FTBER LASE Boston '88, Boston, MA, September 6-10, 1988

September 9, 1988

This is a preprint of a paper intended for publication in a journal or proceedinga. Since chauges may be made befole publication, this preprint is made available with the understanding that it will not be cited or reproduced without the permission of the author.

\section{DISCLAIMER}

This report was prepared as an account of work sponsored by an agency of the I/nited States Government. Neither the United States Government nor any agency thereof, nor any of their employees, makes any warranty, express or implied, or assumes any legal liability or responsibility for the accuracy, completeness, or usefulness of any information, apparalus, product, or process disclosed, or represents that its use would not infringe privately uwned rights. Reference herein to any specific commer.jal producl, process, or service by trade name, trademark, manufacturer, or otherwise does not necessarily constitute or imply its endorsement, recommendation, or favoring by the United States Fovernment or any agency thereuf. The views and opinions of authors expressed t.ercin do not necessarily stute or reflect those of the United States Government or anj agency thereof. 


\title{
A fast radiation-to-coherent light converter
}

\author{
C. L. Wang, J. E. Flätley, and P. H Stewart \\ University of California, Lawrence Livermore National Laboratory \\ P.O. Box 808, Livermore, CA 94551 \\ N. Bar-Chaim, K. Y. Lau, and I. Ury \\ Ortel Corporation \\ 2015 West Chestnut Street, Alhambra, CA 91803
}

\author{
R. M. Baltrusaitis, T. J. Davies, B. A. Davis, S. G. Iversen, B. R. Marshall, M. C. Thomas, and P. A. Zagarino \\ EG\&G, Inc. \\ P.O. Box 98 , Goleta, CA 93017
}

\begin{abstract}
We have developed a radiation-to-coherent light converter (RCLC) with a monolithically integrated semiconductor chip that consists of a chromium-doped GaAs photoconductor detector and a GaAlAs laser diode. When irradiated, the electric pulse generated by the photoconductor detector modulates the laser diode, which has been biased above the lasing threshold, thus converting a radiation pulse to an electric pulse and then to a light pulse. The laser pulse is then transmitted to a fast recorder through a high-bandwidth optical fiber. In the absence of a single-step $x$-ray pumped laser, our converter appears to be the first integrated device that can efficiently convert $x$-ray flux into coherent light. This device has been tested successfully with the 50-ps electron beams of a $17-\mathrm{MeV}$ linear accelerator and with 50 -ns $\mathrm{x}$-ray pulses from a Z-pinch plasma source.
\end{abstract}

\section{INTRODUCTION}

For high-speed $x$-ray spectroscopy or imaging with $\leq 200$-ps rsolution that requires transporting analog signals to a fast recorder at a distance, the development of an array of RCLCs appears necessary. By converting radiation to coherent light, one can take advantage of the high transmission bandwidth of the optical fiber. Optical signals can be transmitted over long fibers (on the order of kilometers) with a multigigahertz bandwidth that is virtually impossible to obtain with a conventional electric pulse via coaxial cables.

Figure 1 depicts the concept of a high-bandwidth, radiation-detection, optical transport and recording system. As a first step toward developing such a system, we have designed, built, and tested a single element of the RCLC.

\section{RADIATION-TO-COHERENT LIGHT CONVERTER (RCLC)}

The feasibility of making an RCLC became clear after our recent development of ultrafast GaAsphotoconductor radiation detectors. ${ }^{1}$ By the use of proper radiation damage for generating crystal deiects that shorten the carrier lifetimes, we obtained GaAs detectors with 30-ps resolution. With chromium doping, we can also obtain $\mathrm{GaAs}$ detectors of 200 -ps resolution. Note that this semi-insulating GaAs is routinely used as the substrate of the high-speed GaAsAs laser diode. ${ }^{2}$ This opens up the possibility of building a monolithically integrated RCLC.

Our RCLC is a monolithically integrated semiconducor chip that consists of a chromium-doped GaAs photoconductor detector and a GaAlAs laser diode. One part of the $\mathrm{GaAs:Cr}$ photoconductor has two electrodes and functions as a high-speed radiation detector, while the other part of this semi-insulating crystal serves as the substrate for the GaAlAs laser diode. These two parts are connected in series, as shown in Fig. 2. The top part is a photoconductor detector, shown as a variable resistor, while the bottom part is a high-speed laser diode.

Nominally, the laser is biased by injection current beyond the lasing threshold in the linear region. When irradiated, the photoconductor becomes a variable iesistor, allowing extra current to be injected into the cavity, 


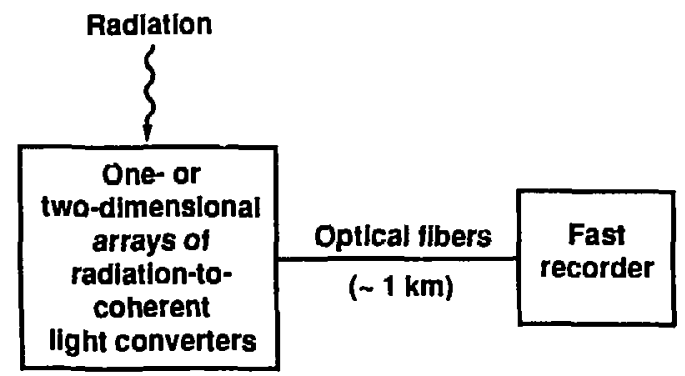

Figure 1. Concept of a multigigahertz radiation-spectrum/image-detection system for long-distance analog-signal transport.

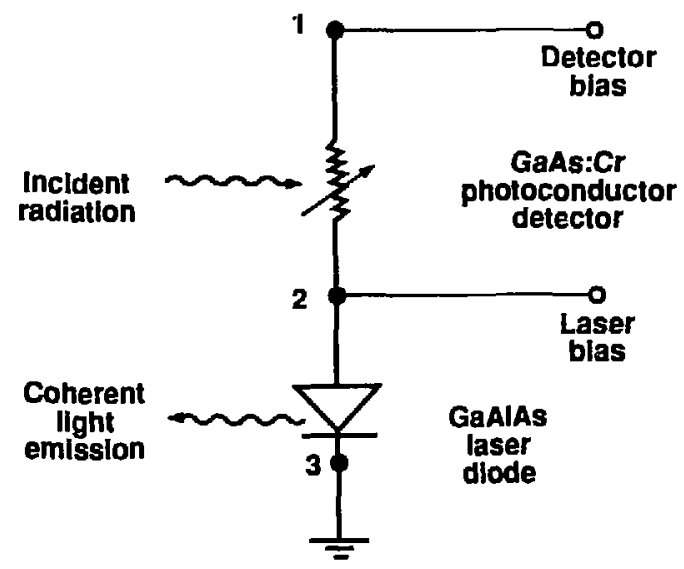

Figure 2. Circuit diagram of an integrated detector-laser chip as a radiation-to-coherent light converter (see Fig. 3 for explanation of points 1, 2, and 3).

thus modulating the laser output. The photoconductor is operated in a linear region wherein the induced current is proportional to the energy of the absorbed radiation. In other words, the modulated optical signal is proportional to the radiation pulse. Our RCLC is thus a two-step device that converts a radiation pulse to an electric pulse and then to a light pulse.

\section{STRUCTURE AND FUNCTIONS}

Figure 3 shows the structure of our integrated detector-laser transmitter. Note the diagram is not to scale (see Fig. 4 in scale). The area between electrodes 1 and 2 is sensitive to radiation. The connection between electrode 2 and the $p$ side of the laser is done at the wafer level (i.e., without wire bond).

The GaAs detector is simply a bulk photoconductive crystal with two ohmic contact electrodes. Electrons and holes created by the incident radiation are collected by the electrodes, generating an electric pulse. This detector is made in a stripline geometry, assuring a fast rise time. Its decay time depends on the carrier lifetimes, which can 


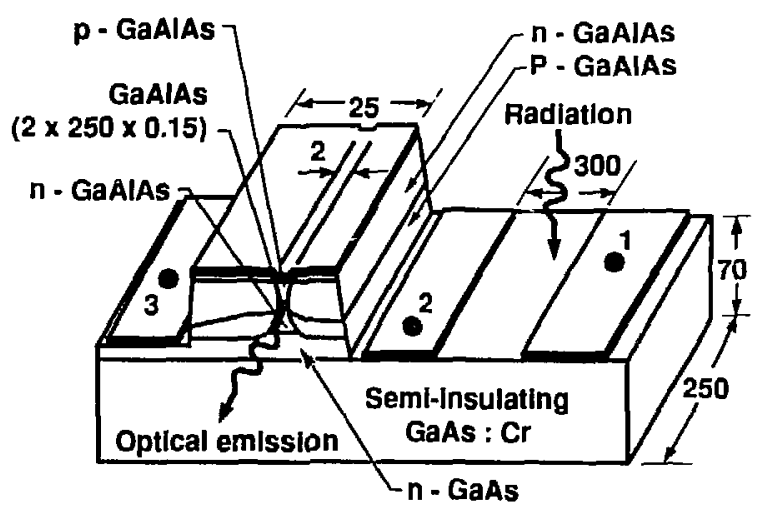

Note: Not to scale

Unit: $\mu \mathrm{m}$

Figure 3. Schematic of the integrated radiation detector-laser transmitter. Points 1,2 , and 3 are gold electrodes. The $\mathrm{p}$ side of the laser and electrode 2 are connected at the wafer level (no wire bond).

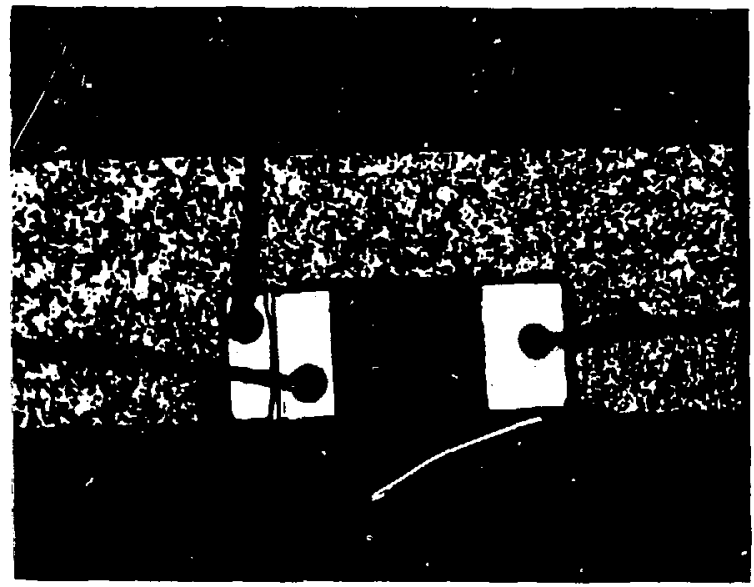

Figure 4. Photograph of the fabricated radiation-to-coherent light converter.

be controlled by radiation treatment or, to some extent, by impurity doping (see Ref. 1 for mcre information on the photoconductor detector).

The high-speed GaAlAs laser is the state-of-the-art "buried heterostructure window laser." so named because numerous heterojunctions (junctions of different material compositions) bury one junction after another. These heterojunctions confine electrons and light to certain regions where they can interact efficiently for maximum conversion of electrons to light. The "window" refers to the narrow transparent layer of unpumped GaAlAs at the end region near the crystal facets for protection of the laser mirror, thereby allowing a high optical-power capability. The modulation speed of this device can reach $10 \mathrm{GHz}$ (see Ref. 2 for further details on the fast laser diode). 
Figure 4 is a photograph of the fabricated device mounted on a copper block as a heat sink. Wire bonds at points 1, 2, and 3 on the electrodes connect to power supplies and ground. The device is $750 \mu \mathrm{m}$ long and $250 \mu \mathrm{m}$ wide. After the chip was fabricated, the photoconductor detector and the laser were checked separately: the detector with a short optical pulse to confirm its function, and the laser by measuring the light output as a function of injection current (see Fig. 5). The low lasing threshold $(<20 \mathrm{~mA})$ alleviates the heat-sink problem usually associated with these devices. The optical pure, single, longitudinal mode of the optical spectrum is important for fiber-optic transmission because it eliminates material dispersion, thereby allowing high-bandwidth and longdistance transmission.
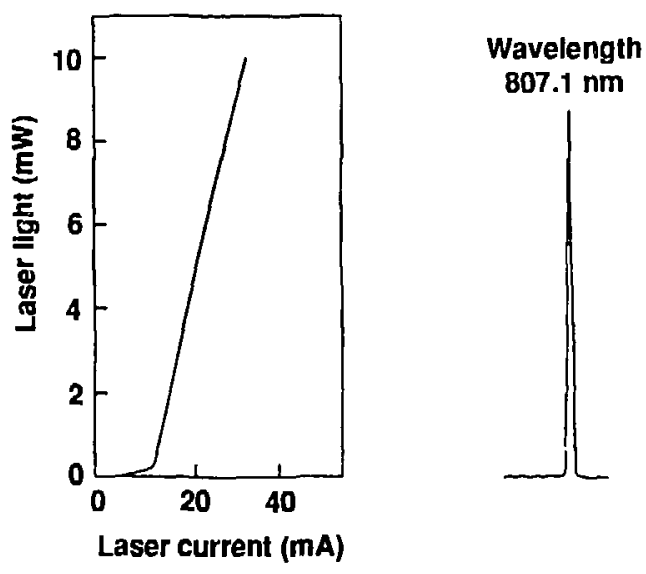

Figure 5. Laser light output as a function of injection current and the lasing wavelength.

\section{EXPERIMENTS}

To test our RCLC with 16-MeV electrons and $\mathrm{keV} \times$ rays, we mounted the chip inside an rf shield (see Fig. 6). The if shield is important for reducing the if noise in the linear accelerator environment and the EMP background near the Z-pinch plasma source. A radiation-entrance hole is provided directly above the chip. The package is mounted on a flange for a low-energy $\mathrm{x}$-ray experiment in a vacuum. For $16-\mathrm{MeV}$ electrons, the device is operated in air. The laser emits at $90^{\circ}$ to the incident radiation. The light output is collimated with an aspheric lens with a 4.6- $\mathrm{mm}$ focal length and a $0.55 \mathrm{NA}$ and focused onto a $50-\mu \mathrm{m}$ fiber with another lens. The detector and laser biases are supplied through semirigid coaxial cables, which are brought to within about $3 \mathrm{~mm}$ of the chip to minimize the impedance mismatch.

The impulse response of our RCLC to 50 -ps electron beam is shown to be 170 ps FWHM (see Fig. 7 ). The laser output was transmitted with an optical fiber to a fast PIN diode and analyzed with a sampling scope. We also used a coupling capacitor to monitor the impulse risponse of the $\mathrm{GaAs}: \mathrm{Cr}$ detector to the electron beam. This turned out to be 200 ps FWHM, somewhat longer than the laser output. Preliminary investigation indicates that a possible low-impedance reflection may exist in the laser bias lead, causing the shortening of the laser pulse.

For the detection of a low-energy $x$-ray pulse from a $Z$-pinch plasma, the RCLC was operated in a vacuum. We placed an $x$-ray filter in front of the radiation-entrance hole to block the visible light from the plasma source. Figures $8(a)$ and (b) show, respectively, the $x$-ray pulse observed with the $\mathrm{GaAs}: \mathrm{Cr}$ detector and the laser output detected by a PIN diode through an optical fiber. It is seen that the fidelity is remarkable. There appears a little more noise in the coaxial cable (a) than in the optical fiber transmission (b). 


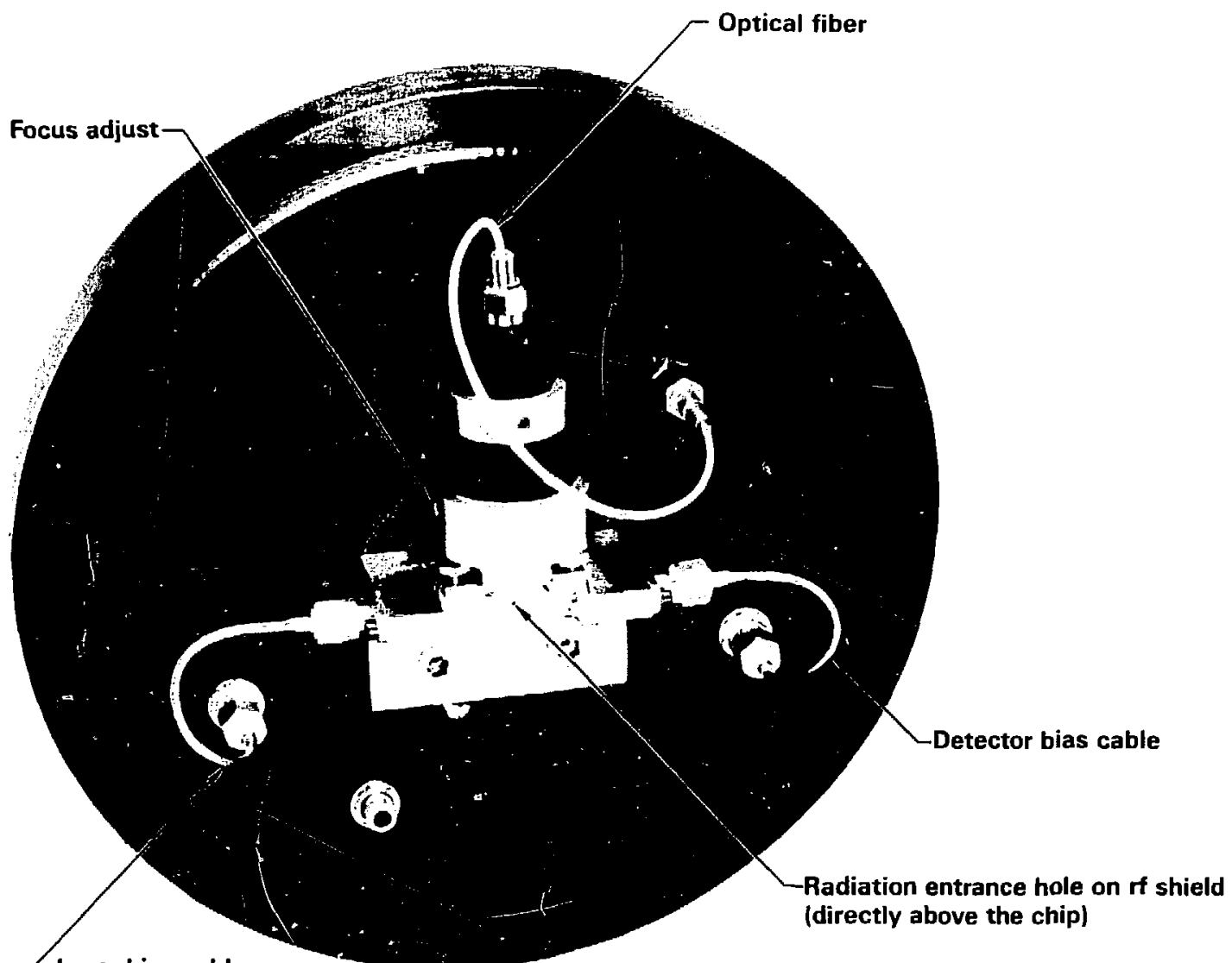

$\angle$ Laser bias cable

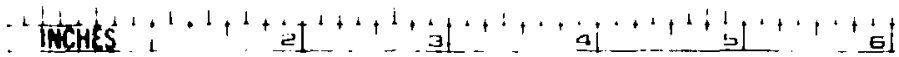

Figure 6. The radiation-to-coherent light converter mounted inside an $\mathrm{rf}$ shield. The output light is lens-coupled to an optical fiber.

The correlation between the pulse heights from the GaAs detector and the laser output is plotted in Fig. 9. It is linear for the range covered here. We will cover a larger range in a future experiment.

\section{DISCUSSION}

We are aware of concern regarding the possible radiation damage to the laser diode in the integrated device. In a case witi highly penetrating radiations, such as gamma rays or high-energy electrons, the energy loss in the thin laser cavity is so smail that no damage is expected. No radiation damage was observed throughout the experiment with the 16- $\mathrm{MeV}$ electron beam. In the case of low-energy $x$ rays, the electrode can be made thick enough to keep $x$ iays from reaching the laser cavity, thus preventing radiation damage. No radiation damage was observed during our experiment with $x$ rays. 


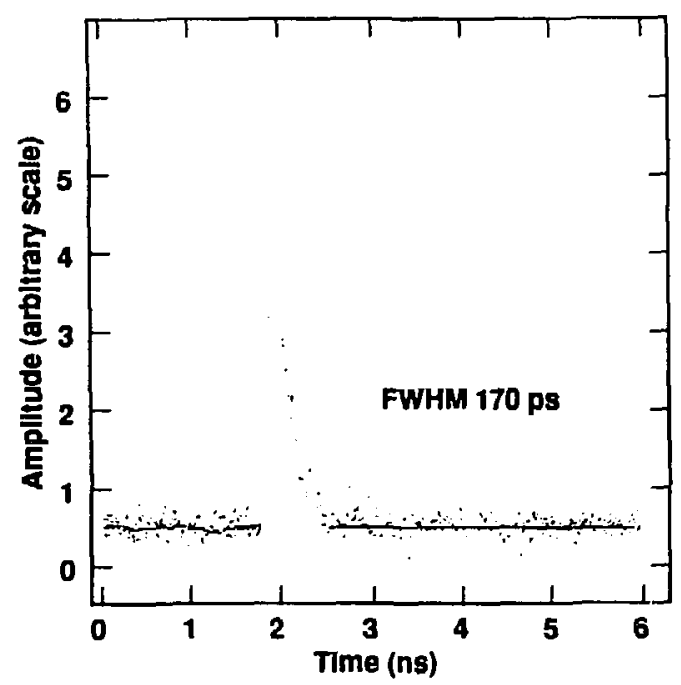

Figure 7. Impulse response of the radiation-to-coherent light converter to 50-ps electron beam.

(a)

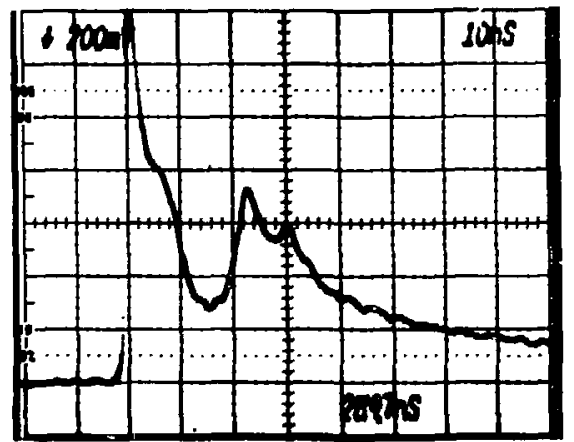

(b)

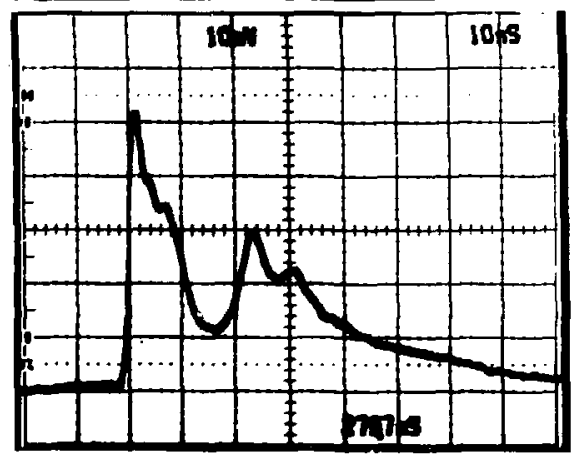

Figure 8. Comparison of the $x$-ray pulse observed with the GaAs:Cr detector (a) and the laser output detected by a PIN diode (b). A good fidelity is observed.

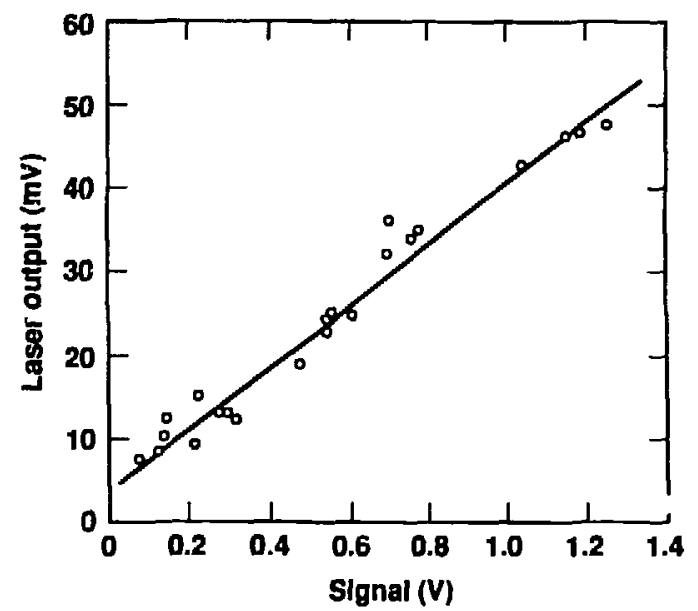

Figure 9. Linear correlation between pulse heights from the GaAs: $\mathrm{Cr}$ detector and output from the laser. 


\section{CONCLUSION}

Our RCLC functioned reasonably well from the first attempt. In the absence of a single-step x-ray pumped laser, it appears to be the first integrated device that can efficiently convert $x$ rays or other radiations into coherent light. It opens up the possibilities for one- or two-dimensional arrays and large-scale integration. However, a nontrivial effort will be required in optical fiber pigtailing for stch applications.

\section{ACKNOWLEDGMENT}

Work was performed under the auspices of the U.S. Department of Energy by the Lawrence Livermore National Laboratory under contract No. W-7405-Eng-48.

\section{REFERENCES}

1. C. L. Wang, M. D. Pocha, J. D. Morse, M. S. Singh, and B. A. Davis, Proc. SPIE 836, 15 (1987).

2. K. Y. Lau, N. Bar-Chaim, I. Ury, and A. Yariv, Appl. Phys. Lett. 45, 316 (1984). 